\title{
Infrared Spectroscopy of Human Tissue. V. Infrared Spectroscopic Studies of Myeloid Leukemia (ML-1) Cells at Different Phases of the Cell Cycle
}

\author{
SUSIE BOYDSTON-WHITE, ${ }^{1}$ TAMARA GOPEN, ${ }^{2}$ SANDRA HOUSER, ${ }^{2}$ JILL BARGONETTI, $^{2}$ MAX DIEM $^{1}$ \\ ${ }^{1}$ Departments of Chemistry and Biochemistry, City University of New York, Hunter College, \\ 695 Park Avenue, New York, New York 10021 \\ 2 Department of Biological Science, City University of New York, Hunter College, \\ 695 Park Avenue, New York, New York 10021
}

Received 19 October 1998; revised 24 November 1998; accepted 10 February 1999

\begin{abstract}
Infrared spectra of myeloid leukemia (ML-1) cells are reported for cells derived from an asynchronous, exponentially growing culture, as well as for cells that were fractionated according to their stage within the cell division cycle. The observed results suggest that the cells' DNA is detectable by infrared spectroscopy mainly when the cell is in the S phase, during the replication of DNA. In the G1 and G2 phases, the DNA is so tightly packed in the nucleus that it appears opaque to infrared radiation. Consequently, the nucleic acid spectral contributions in the G1 and G2 phases would be mostly that of cytoplasmic RNA. These results suggest that infrared spectral changes observed earlier between normal and abnormal cells may have been due to different distributions of cells within the stages of the cell division cycle. (c) 1999 John Wiley \& Sons, Inc. Biospectroscopy 5: 219-227, 1999
\end{abstract}

Keywords: infrared spectroscopy; myeloid leukemia cells; cell division cycle

\section{INTRODUCTION}

During the past decade, progress in infrared spectroscopic instrumentation has increased the sensitivity of the measurement to such a degree that

Correspondence to: M. Diem (mdiemhc@aol.com)

Contract grant sponsor: Department of Defense Career

Development Award (to J.B.); contract grant number: DAMD 17-94-J-4030.

Contract grant sponsor: American Cancer Society (to J.B.); contract grant number: CN-140.

Contract grant sponsor: National Science Foundation (to J.B.); contract grant number: MCB-97 22262.

Contract grant sponsor: American Cancer Society Research Opportunity Grant (to M.D.); contract grant number: ROG 99-119-01.

Contract grant sponsor: Hunter College Presidential New Research and Teaching Initiative Award (to M.D.).

Biospectroscopy, Vol. 5, 219-227 (1999)

(c) 1999 John Wiley \& Sons, Inc.

CCC 1075-4261/99/040219-09 the acquisition of excellent spectral data from a single mammalian cell is possible. Consequently, several research groups have been involved in applying infrared spectroscopy and infrared microspectroscopy to a number of problems in biomedical research, with the particular emphasis on detecting cellular abnormality spectroscopically. For example, we have shown ${ }^{1}$ that in biopsied sections of cervical tissue diagnosed to be normal, dysplastic, and neoplastic by standard histopathological methods, the infrared spectra collected for pixels measuring ca. $50 \times 50 \mu \mathrm{m}$ on edge can distinguish the state of health of the tissue, although the differences between healthy and diseased tissues are quite subtle and certainly much smaller than those reported erroneously for exfoliated cervical cells. ${ }^{2}$ However, an interpretation of the spectral differences between 
normal and abnormal tissues was not possible at the time. ${ }^{3,4}$

Similar efforts on exfoliated cervical individual cells may be possible as well, although early attempts $^{2,5,6}$ in this direction were confounded by the enormous heterogeneity ${ }^{3,4,7}$ in the composition of the cell exfoliates that makes any cursory correlation between spectral features and a cytological diagnosis impossible. Furthermore, it appears that the high correlation between infrared results and pathological standards in some of the early studies was fortuitous.

Thus, we have initiated a research program that is aimed at understanding the spectral features of cells, and the variations in spectral features due to a variety of causes. Early results from these studies demonstrate that for cell samples of high homogeneity, spectral changes due to a number of causes, such as drug treatment, apoptosis, and so forth, can be discerned. In this publication, we report spectral differences between exponentially growing cultured cells that were separated according to the phases of the cell cycle. The detailed interpretation of these results leads to a clearer understanding of the origins of the spectral differences between normal and abnormal cells.

A different approach of applying infrared spectroscopy for the detection of cancer was pioneered by Malins and coworkers. ${ }^{8}$ They have reported spectral differences between DNA isolated from normal and cancerous tissues from a number of organs, and have reported similar patterns in the spectral changes. It is interesting to note that the spectral changes reported below for entire cells parallel the experimental results reported by $\mathrm{Ma}-$ lins. We shall address this aspect later in the discussion section.

\section{BACKGROUND}

\section{Summary of Infrared Spectroscopic Principles}

Since this publication is intended for audiences of varied backgrounds, a brief summary of spectroscopic principles, as well as some aspects of cellular development will be presented here.

In infrared spectroscopy, the attenuation of the intensity of a beam of infrared light upon passing through a sample is measured. This attenuation is caused by the interaction of the light with the vibrational transitions of the sample molecules. These absorptions of infrared light, when plotted against the wavelength of the light, produce a unique fingerprint pattern of the molecules encountered by the beam of light. Such fingerprint patterns for a number of cellular components are shown later in this article. In addition, one can assess the degree of packing of certain cellular components, from the infrared spectral patterns, that is, we have postulated before that a nucleus of a quiescent cell is optically so dense, that it may not transmit any of the incident infrared radiation. ${ }^{3}$ Consequently, infrared spectroscopy may be used to monitor nuclear processes which result in significant changes in the packing of the constituent molecules. This aspect will be elaborated upon below.

In infrared microspectroscopy (also referred to as infrared microscopy) the infrared beam is passed through and focused by an infrared microscope that allows infrared spectral data to be collected from microscopic particles, such as single cells, or pixels of tissue the size of a cell.

The variety of molecules found in a human cell is so staggering, the unambiguous assignment of the infrared spectra of a cell's constituents is not possible, particularly since different proteins, in general, have similar vibrational spectra. However, we and others have shown that changes in molecular composition can be observed and interpreted reliably. ${ }^{3,9}$ For example, different protein/ nucleic acid ratios, or the overexpression of structural proteins, can be monitored via infrared microspectroscopy.

\section{Cell Biological Aspects}

The cells utilized in this study are cultured myeloid leukemia (ML-1) cells. These cells are cancerous, partially differentiated leukocytes that can be grown in cell culture indefinitely. Such cancerous cells undergo continuous cell division, and therefore, the number of cells in a culture of ML-1 cells doubles in a specific time period (for ML-1 cells, this period is about $24 \mathrm{~h}$ ). Such a culture is referred to as "exponentially growing." A typical sample of cells derived from such a culture is highly homogeneous; the only heterogeneity being due to the fact that cells in an exponentially growing culture exist at different stages of their 24-h cell division cycle.

The cell division cycle can be divided into four distinct major stages referred to as the Gap 1 (G1), Synthesis (S), Gap 2 (G2), and Mitosis (M) phases. In exponentially growing cell culture, the proportion of cells found in a given phase of the 
cycle is determined by the relative length of these stages, which is about $10 \mathrm{~h}$ for the $\mathrm{S}$ phase, $8 \mathrm{~h}$ for G1, $6 \mathrm{~h}$ for G2, and less than an hour for the M phase. ${ }^{10}$

In order to isolate cells at given stages of their cycles, cell cultures can be synchronized, that is, manipulated such that all of the cells in the culture are undergoing each of the phases simultaneously. Alternatively, exponentially growing cells can be separated into fractions of good phase homogeneity by the process of centrifugal elutriation. ${ }^{11}$ In elutriation, cells are centrifuged against a flow of liquid pumped to oppose the centrifugal force. In this fashion, separation by size and density is achieved. ${ }^{12-14}$ Cells at different stages of the cell cycle can be separated by elutriation because their size and density increase between G1, $\mathrm{S}$, and $\mathrm{G} 2$ phases.

We used fluorescence-activated cell sorting $(\text { FACS })^{15,16}$ to establish the identity of the cell fractions collected from the elutriation experiment. FACS is a flow cytometry method in which individual cells can be sorted by various attributes by staining them appropriately. Here, cells were stained with a dye that specifically binds to, and becomes fluorescent, upon binding DNA. The FACS analysis consists of measuring the fluorescent intensity of the complexed dye, which is proportional to the DNA content of the cell, from which the phase of the cell cycle for each cell can be established. The combination of centrifugal elutriation and FACS analysis provided populations of highly homogeneous cells for which cell-cycle information is known.

\section{EXPERIMENTAL PROCEDURES}

The conditions for cell growth, and elutriation and results from FACS analysis have been reported elsewhere ${ }^{17}$ Live cells obtained after elutriation, or from an exponentially growing cell culture, were deposited onto a infrared transparent polyethylene film, fixed with ethanol for $15 \mathrm{~s}$, and dried for spectral data acquisition.

The deposition of the cells on the substrate is extremely critical, since the formation of multilayers more than a few cell layers thick may produce artifacts in the infrared spectra. These artifacts arise because a clump of cells may exhibit absorbances in the amide I region in excess of 2 OD units. Such high absorbances may cause deviations from Beer-Lambert's law, and consequently, produce artificial amide I / amide II ra- tios. The problem is compounded by the fact that the amide I absorption, averaged over a large area of the substrate, may appear reasonable (i.e., $<1 \mathrm{OD}$ ) if the substrate is populated very unequally; that is, if there are areas devoid of cells and areas with very dense cell clumps.

Prolonged treatment of the cell with ethanol will denature the cellular proteins, and will actually lyse and fuse adjoining cells. However, a fast washing with cold $\left(0^{\circ} \mathrm{C}\right)$ ethanol, lasting less than $30 \mathrm{~s}$, does not produce any discernible spectral changes, and fixes the cells satisfactorily. Thus, the following method for sample preparation was employed. Cells were centrifuged, washed repeatedly, and resuspended in isotonic saline. About $0.1 \mathrm{~mL}$ of such a suspension, containing ca. $10^{5}$ cells/mL, was deposited onto a previously wetted polyethylene membrane such that the sample spot did not exceed about $10 \mathrm{~mm}^{2}$. The samples prepared in this fashion were inspected visually, using visible light microscopy. Only samples consisting of sparse monolayers of cells were used for spectroscopic measurements.

Macroscopic collection of infrared absorption spectra was performed using a MIDAC, Inc., Fourier transform infrared (FTIR) spectrophotometer (Model M), by coadding between 20 and 50 interferograms at a resolution of $2 \mathrm{~cm}^{-1}$. In this mode, the infrared beam samples an area of about 10 $\mathrm{mm}^{2}$ containing about $10^{4}$ cells. These macroscopically acquired spectra represent an average of the spectral features of all cells in the infrared beam. However, since the elutriated samples are presumed to be highly homogeneous, these results are representative of the cells in their particular division stage.

Microscopic data acquisition was performed on a Bruker Optics, Inc., Vector 22 FTIR instrument, coupled to an Irscope2 microscope. This instrument permits microscopic data acquisition of objects as small as $15 \mu \mathrm{m}$ in diameter. Generally, cells were selected visually before spectral data were collected microscopically using a $15 \mu \mathrm{m}$ aperture. Depending on the selected window size, between 100 and 500 interferograms at $2 \mathrm{~cm}^{-1}$ resolution were collected and coadded. The raw data obtained after Fourier transform from either of the two spectrometers were converted to a universal data format for further data manipulation. Data manipulation included background correction, smoothing, and-in the case of microscopic data-correction for atmospheric water background. All infrared data are reported as absorption spectra, with ordinate intensities of about 


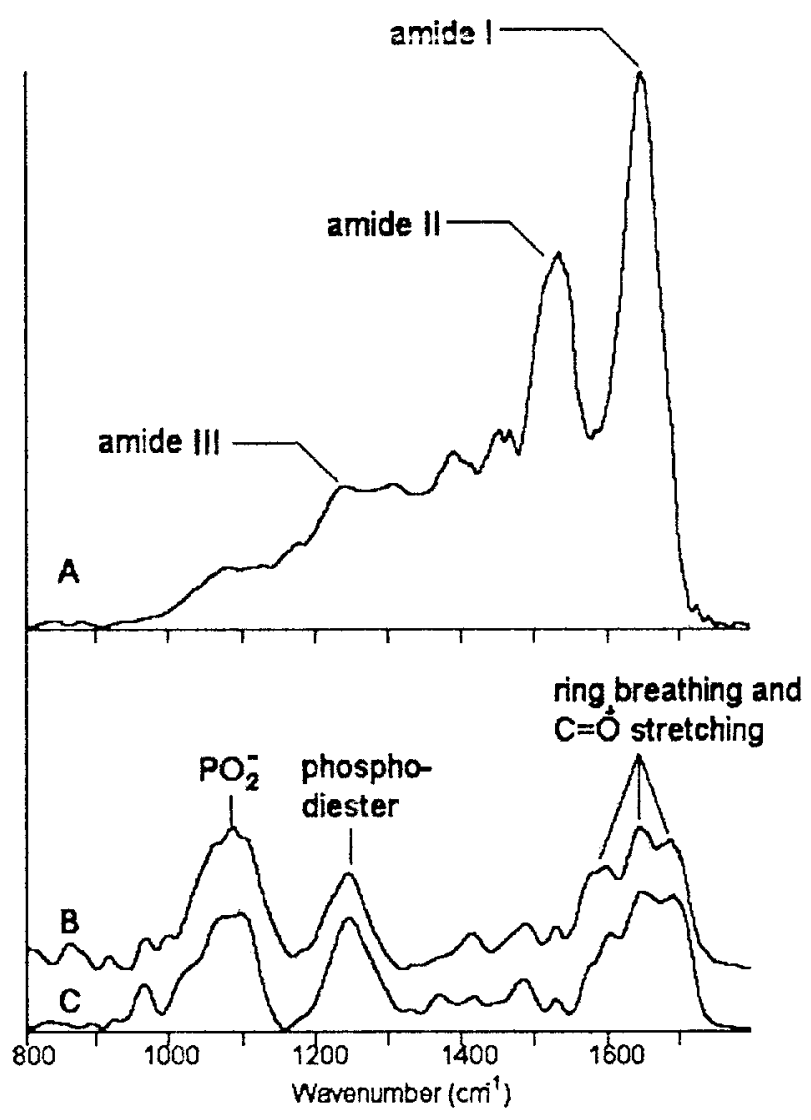

Figure 1. Infrared absorption spectra of cellular components (collected as dried films). Trace A: protein; trace B: r-RNA; trace C: DNA.

0.03 and 0.4 absorbance units at $1655 \mathrm{~cm}^{-1}$ for micro- and macroscopic data acquisition, respectively.

Carefully acquired macroscopic and single cell microscopic data require only minimal background correction. Data shown in Figures 1-3 never exhibited more than a $5 \%$ deviation due to scattering or other baseline artifacts. We use a background correction method modeled after the "rubber band algorithm" that is part of the Bruker Opus ${ }^{18}$ software. For the spectra shown here, this algorithm resulted in a straight line correction between 800 and $1800 \mathrm{~cm}^{-1}$.

\section{RESULTS}

\section{Spectra from Samples of Exponentially Growing ML-1 Cells}

Figure 1 shows the infrared absorption spectra of some of the major cellular components, protein,

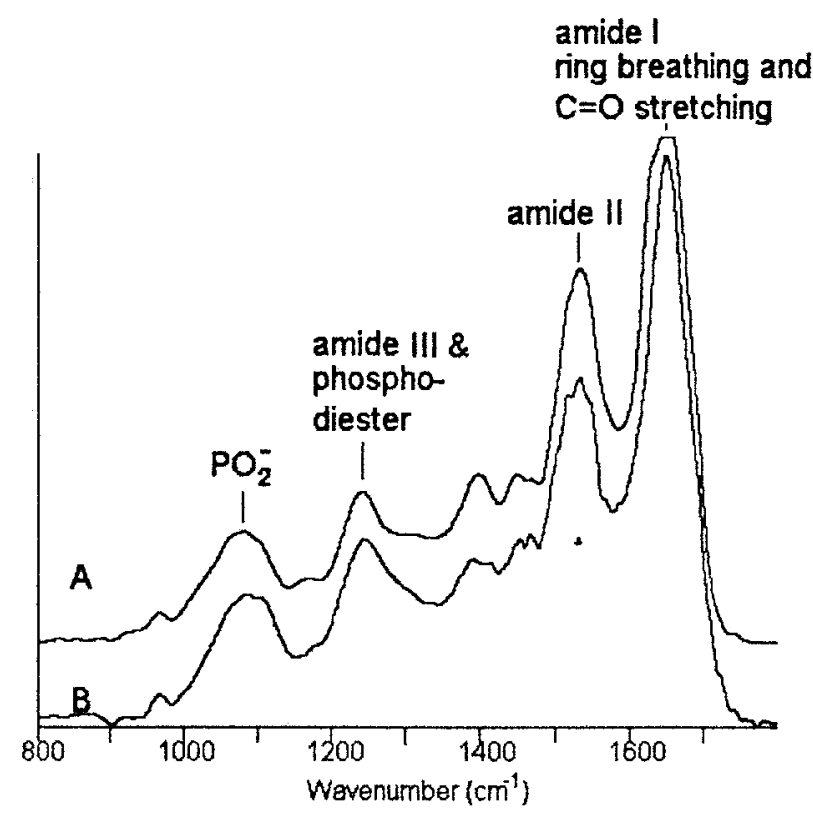

Figure 2. Comparison of a superposition of protein/ RNA/DNA spectra (trace A) with infrared absorption spectra of ML-1 cells (trace B).

RNA and DNA, collected from dried films on $\mathrm{BaF}_{2}$ substrates. Protein spectra, in general, are dominated by the amide I band at ca. $1650 \mathrm{~cm}^{-1}$ that

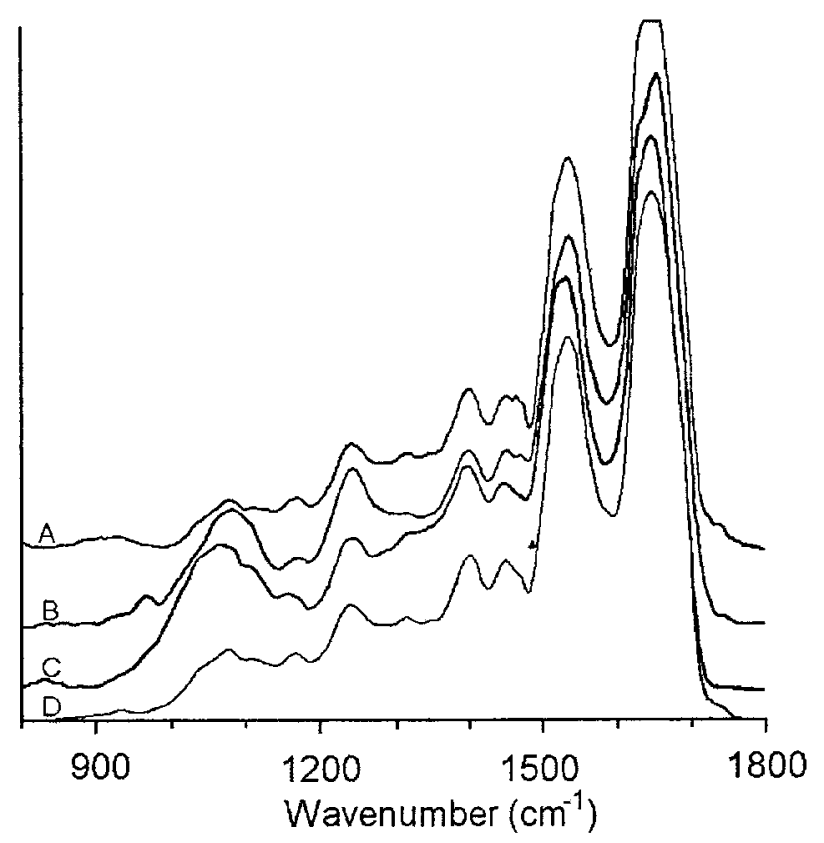

Figure 3. Infrared absorption spectra of ML-1 cells separated into fractions by centrifugal elutriation. Trace A: G1 phase (fraction 5); trace B: S phase (fraction 11); trace C: S phase (fraction 11); trace D: G2 phase (fraction 15). 
is primarily associated with the stretching motion of the $\mathrm{C}=\mathrm{O}$ group ${ }^{19}$ [Fig. 1(A)]. This peak is sensitive to the environment of the peptide linkage, and also depends on the protein's overall secondary structure. The amide II vibration, mainly a coupled $\mathrm{C}-\mathrm{N}$ stretching and a $\mathrm{CNH}$ deformation coordinate, occurs at ca. $1530 \mathrm{~cm}^{-1}$. Weaker protein vibrations include the amide III peak at ca. $1245 \mathrm{~cm}^{-1}$, and a number of side chain vibrations in the 1310,1390 , and $1450 \mathrm{~cm}^{-1}$ range.

Nucleic acids exhibit absorption peaks between 1580 and $1700 \mathrm{~cm}^{-1}$ due to the aromatic base breathing and $\mathrm{C}=\mathrm{O}$ stretching vibrations [Fig. 1 (B), (C)]. ${ }^{20}$ A triad of peaks, due to ribose $\mathrm{C}-\mathrm{O}$ and the ionized $\mathrm{PO}_{2-}$ group stretching vibrations occurs in DNA at 1060, 1084, and $1095 \mathrm{~cm}^{-1}$ with nearly equal intensities. Further DNA peaks are observed at 965 and $1230 \mathrm{~cm}^{-1}$ (the phosphodiester vibrations). In RNA, the peak at $1085 \mathrm{~cm}^{-1}$ is stronger than the two other peaks in this triad, and forms a distinct "nose." Furthermore, the intensity ratio of this triad to the phosphodiester peak that occurs in RNA at $1245 \mathrm{~cm}^{-1}$ is about 1 : 0.7 , whereas it is closer to $1: 1$ in DNA.

DNA spectra are very sensitive to the degree of hydration of the polymer. The spectrum for a hydrated film of DNA exhibits three sharp peaks at $967,1054,1090$, and $1225 \mathrm{~cm}^{-1}$; in a dried film of DNA, the peak at $1054 \mathrm{~cm}^{-1}$ occurs at higher wavenumber $\left(1067 \mathrm{~cm}^{-1}\right)$ and much enhanced intensity. Finally, in a sample of DNA precipitated from ethanol, the $1067 \mathrm{~cm}^{-1}$ peak has nearly the same intensity as the peak at $1090 \mathrm{~cm}^{-1}$. These observations are consistent with our understanding that DNA may undergo a phase transition from the B- to the A-type form under dehydrating conditions. ${ }^{21}$ The A-Type DNA structure is very similar to the right-handed RNA double helix. Therefore, it is not surprising that the sharp spectrum of hydrated DNA assumes the broad, RNAlike infrared spectrum upon dehydration.

In Figure 2, a comparison of spectral patterns observed for exponentially growing ML-1 cells (Trace B), and a spectral superposition of RNA/ DNA and protein spectral features (Trace A) is shown. This figure demonstrates that the observed spectra for the ML-1 cells can be reproduced reasonably well by a superposition of the component spectra shown in Figure 1. The extensive overlap between nucleic acid and protein spectra does not permit an easy estimate of the absolute DNA, RNA, and protein contributions.

The peak at $1245 \mathrm{~cm}^{-1}$ (marked amide III / phosphodiester) is quite intense in both traces

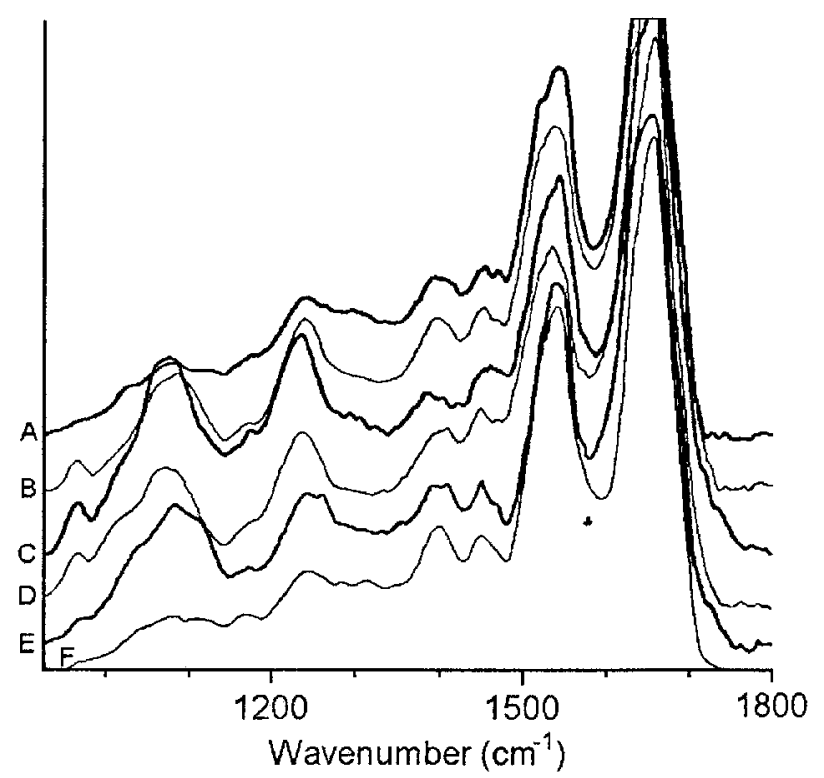

Figure 4. Infrared microspectra of individual ML-1 cells separated into fractions by centrifugal elutriation: (A) G1 phase; (B-D) early, middle, and late S-phase; (E) S/G2 interface; (F) G2 phase.

shown in Figure 2, since it is a superposition of the protein amide III and the nucleic acid phosphodiester peaks. Thus, in cells and tissues, this peak is observed with higher intensity than the $\mathrm{PO}_{2}^{-}$triad of peaks. The amide I peak, and the valley between the amide I and II peaks, contain vibrational intensities from both the protein $\mathrm{C}=\mathrm{O}$ and the nucleic acid $\mathrm{C}=\mathrm{O}$ stretching vibrations; consequently, only the protein amide II peak occurs without interference from nucleic acid peaks.

\section{Cell Cycle Dependent Spectra of ML-1 Cells}

Figures 3 and 4 show infrared absorption spectra of cells separated into fractions according to the phases of the cell cycle by centrifugal elutriation. These fractions were subsequently analyzed by FACS and identified by DNA content as to the stage of the cell cycle. ${ }^{17}$ The data in Figure 3 were collected macroscopically; that is, each spectrum shown represents the average of an unknown number of cells on the sample substrate. Since the cells were separated into fractions and elutriation, we may assume that each spectrum is fairly representative of cells at a given stage in the cell cycle.

Data in Figure 4 were obtained from a different elutriation experiment, and they represent micro- 
scopically acquired (single cell) data. The spectra shown were obtained by depositing cells from one

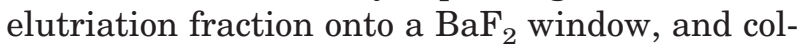
lecting spectra from between 10 and 50 individual cells selected visually. Although the cells on one window were all from the same elutriated fraction, we found still a small heterogeneity of the spectra, particularly in the later phases. These single cell spectra were analyzed for the variations in DNA and RNA spectral contribution by a variety of quantitative methods, including hyperspectral analysis. Results from these studies will be reported at a later date.

A representative spectrum of cells in the G1 phase, collected macroscopically, is shown in Figure 3 , trace A. This spectrum is typical for a cell with low spectral contributions of nucleic acids, and is predominated by protein spectral contributions. The observable nucleic acid features around $1080 \mathrm{~cm}^{-1}$ are typical for RNA spectral features (vide infra). ${ }^{22}$ The spectral variation among the first seven fractions of cells was within experimental reproducibility for macroscopically collected data.

Traces B and C of Figure 3 represents cells from elutriation fraction 8 and 11, respectively, that were found to contain $\sim 90 \%$ of the cells in the $\mathrm{S}$ phase. There is considerable heterogeneity in the $\mathrm{S}$ phase spectra, beginning with a gradual increase of the DNA contribution to the spectra, to a very large DNA contribution shown by Trace B. By fraction 11, the relatively narrow DNA features shown in Trace B (including a prominent $969 \mathrm{~cm}^{-1}$ DNA-specific peak) are replaced by broader features more typical of RNA. Benedetti and coworkers ${ }^{22}$ have shown that variations in the ratio of RNA to DNA spectral contributions may lead to a decline in the spectral detail, and broadening of the peaks centered around 1060 $\mathrm{cm}^{-1}$, comparable to the changes observed between traces $\mathrm{B}$ and $\mathrm{C}$.

Finally, trace D represents one of three fractions of cells found to be predominantly in the G2 phase $(\sim 70 \%)$. The reproducibility of all the late fractions, where most cells are in the G2 phase, was very good. The low-frequency region of the spectrum, similar to the G1-type spectra, is dominated by features attributable to RNA.

Similarly, Figure 4 shows the corresponding data collected microscopically. Trace A shows the absorption spectrum of a cell in the G1 phase. This spectrum is similar to Trace A of Figure 3, and typical for a cell with low spectral contributions of DNA. Traces B, C, D, and E of Figure 4 show spectra obtained from cells corresponding to early, middle, and late $\mathrm{S}$ phase. The spectral changes observed for the single cells parallel those reported above for macroscopically acquired data. There is considerable heterogeneity of the spectra in the $\mathrm{S}$ phase, starting with a gradual increase of the DNA contribution to the spectra to a very large DNA/RNA contribution shown at the $\mathrm{S} / \mathrm{G} 2$ junction (Trace E). Trace F, from a cell in the G2 phase, shows a low-frequency spectral region similar to that of the G1-type spectrum.

Figure 5 shows the levels of homogeneity of the phases used for the infrared studies. In these histograms, the distribution of cells is plotted against their DNA content, as observed by DNA/ probe fluorescence. This distribution is deconvolved computationally into subsets of cells mostly in G1 [Fig. 5(A)], S [Fig. 5(B,C)], and G2 [Fig. 5 (D)] phases.

\section{DISCUSSION}

\section{G1 and G2 Phases}

In the past, several research groups have published infrared spectral differences between normal and cancerous cells and tissues. ${ }^{1-9}$ The prevalent mode of interpretation in these studies, particularly the early ones by Wong et al., ${ }^{2,23}$ was that any spectral changes between pathological diagnoses of normal and abnormal was attributed to cancer. However, we have shown that the heterogeneity of cells in exfoliated samples, in particular, in the case of cervical dysplasia, can lead to spectral changes that correlate reasonably well with the pathological/cytological diagnoses, but that this correlation is totally fortuitous. ${ }^{1,3,4}$

Here, we present results that demonstrate that it is possible, by infrared spectroscopy, to distinguish the different phases through which somatic cells pass during their regular division cycle. Some of the spectral differences observed for the phases of the cell cycle resemble those observed between normal and abnormal exfoliated cells; thus, it appears that many of the spectral features attributed previously to abnormality rather reflect differences in the abundance of cells at different phases of the cell cycle. Thus, the understanding of the origin of the spectral features of cells, and the differences observed between cells separated into fractions according to their cell cycle stages, is of utmost importance for the 


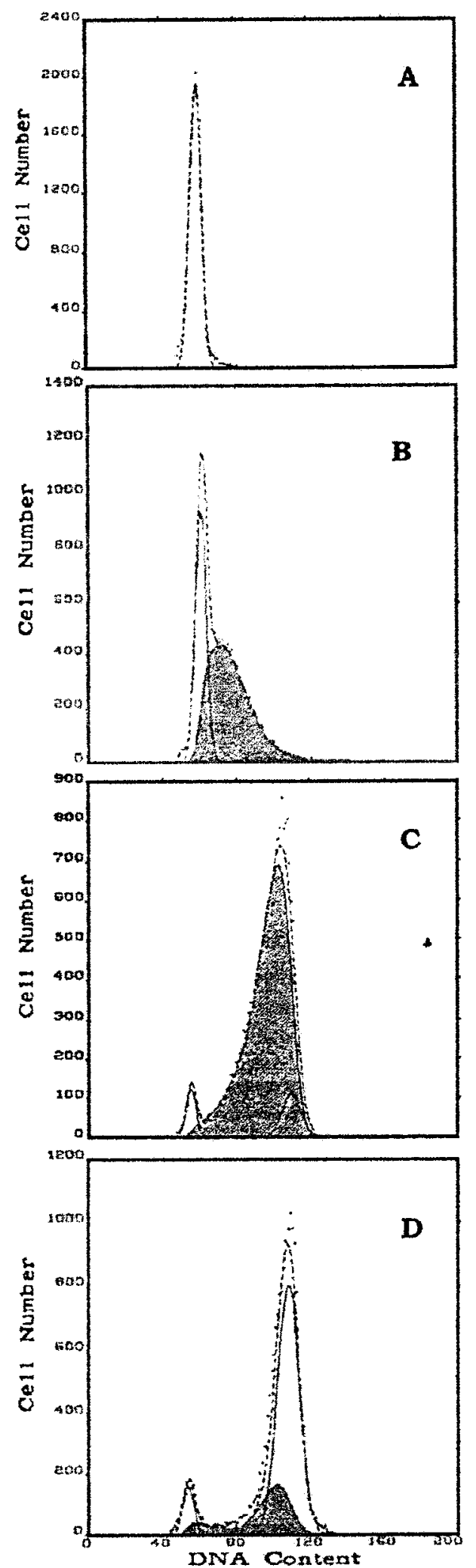

Figure 5. FACS results of (A) fraction 5, (B) fraction 9 , (C) fraction 11, and (D) fraction 15. The graphs show the distribution of cells plotted against the DNA fluorescence. proper interpretation of spectral results for cells and tissues.

One of the most important findings in this study is the extremely low contribution of nucleic acid features to the observed spectra of ML-1 cells in the G1 and G2 phases, and the similarity of spectra of cells in G1 and G2 phases. This similarity is particularly surprising since cells in the G2 phase are tetraploid (four complete copies of chromosomal DNA), while the cells in G1 are still diploid (two complete copies of chromosomal DNA). Thus, one should expect that cells in the G2 phase would show more spectral features due to DNA, which clearly is not the case.

A sample of asynchronous, exponentially growing ML-1 cells, on the other hand, exhibits relatively pronounced DNA/RNA contributions. We propose here a model that accounts for the low visibility of nucleic acids in cases such as cells in the G1 and G2 phases. This model originally was proposed based on the absence of DNA signatures in mature epithelial cells. ${ }^{3}$ The small, condensed nuclei were clearly visible when a cell was examined under visible light microscopy; however, the same cell, examined via infrared microscopy, often failed to exhibit even the slightest spectroscopic signatures of nucleic acids.

For such inactive cells, the nucleus is very small and very well delimited. The concentration of carbonyl groups from the DNA within a nucleus of $5 \mu \mathrm{m}$ diameter is actually quite high, and can be estimated to be on the order of $0.3 \mathrm{M}$, based on the lengths of the human DNA of about $3 \cdot 10^{9}$ base pairs. In addition, carbonyl groups found in the amide linkage of nuclear histones will result in a concentration of carbonyl and peptide linkages of about $1 M$. Using a molar extinction coefficient for a carbonyl group of about $10^{3}[\mathrm{~L} /(\mathrm{mol}$ $\mathrm{cm})$, the optical density of a nucleus was originally estimated to be about 0.5 absorbance units. Adding the effect of the nuclear RNA, found throughout the nucleus and in the nucleolus, we originally postulated that the entire nucleus could be opaque to infrared radiation. ${ }^{3}$

However, the DNA is not distributed uniformly throughout the nucleus; rather, it is wrapped around the histones to form the nucleosomes. Depending on their degree of association (which depends on the phase of the cell cycle), the resulting structures may have a diameter between 30 and $800 \mathrm{~nm}$. Ignoring the effect of RNA, and assuming, for simplicity, a spherical nucleosome with $500 \mathrm{~nm}$ diameter (with the same number of DNA bases pairs), its optical density would exceed 50 . 
Thus, we conclude that the nucleosomes of an inactive nucleus will appear as "black" (i.e., opaque) strings or dots that yield no spectral information from the DNA. Similar arguments may hold for RNA, which occurs in dense particles (nucleolus, ribosome) as well. Thus, the RNA spectral intensities may also depend greatly on the packing of the RNA species.

Therefore, we attribute the "low visibility" of nucleic acids in cells in the (G0), G1, and G2 phases to the fact that they may be packed so tightly that they are not observable in infrared spectroscopy due to very high local absorbances. This is tantamount to saying that parts of the nucleus, of it, appears "black" in the infrared spectrum, and little spectral information of these "black" regions can be obtained.

Other factors confirm this hypothesis. The high spectral detail in the low- frequency (1000-1200 $\mathrm{cm}^{-1}$ ) region of $\mathrm{G} 1$ and $\mathrm{G} 2$ cells suggests that RNA, not DNA, is responsible for the absorptions, based on the band shape analysis reported by Benedetti. ${ }^{22}$ Furthermore, cells with ploidy larger than two (i.e., cells with more than two copies of chromosomal DNA) do not exhibit larger DNA signals than diploid cells. Examples of such cells are the cells in the G2 phase (4-ploid) or the polyploid megakaryocytes reported by Benedetti. ${ }^{24}$ Thus, it appears that not the amount of DNA, but its packing, determines whether or not it is detectable by IR spectroscopy. This fact has not been recognized before, and is necessary for the further exploitation of spectral information from cells and tissues.

\section{S Phase Spectra}

The spectra in the $\mathrm{S}$ phase are quite different from both G1 and G2 phases. During the S phase, the spectral contributions due to DNA increase markedly. This is manifested by an increase of intensity in the 1000 to $1100 \mathrm{~cm}^{-1}$ spectral region, and the loss of spectral details in this region (Fig. 4), which indicates an increased DNA/RNA ratio. $^{22}$ These spectroscopic results suggest that during replication of the DNA in the $\mathrm{S}$ phase, sections of the DNA will become packed less densely, and, consequently, will become detectable by IR radiation. Whereas, in cells in the G0 stage, or nuclei of cells in G1 and G2 phases, the DNA contributes little to the overall spectra.

Asynchronous, exponentially growing ML-1 cells show a spectrum that contains a much larger contribution of DNA versus RNA spectral fea- tures than either G1 or G2 cells. Therefore, we conclude that the average of all cells contains a high proportion of cells in the $\mathrm{S}$ phase. This in agreement with knowledge from cellular biology studies, which have asserted that cycling cells spend more than $50 \%$ of time in the $\mathrm{S}$ phase. Thus, the spectrum of an average ensemble of cells will certainly show DNA features. In addition, spectral features of cells in the M (mitotic) phase may contribute to the averaged spectral features; however, due to the short time cells spend in this phase, its spectral signatures have yet to be established.

The spectral differences between G1 and S phase spectra have previously been associated by some workers with the spectral signature of cancer. The spectral differences observed between canine lymphoma and normal lymphocytes ${ }^{25}$ for example, appear to be due to an altered ratio of S/G1 or S/G2 cells, which in turn, may be due to the fact that abnormal cells which, compared to normal cells, are known to divide more rapidly, and spend more time in the $\mathrm{S}$ phase. On the other hand, the spectral differences could be due to the fact that the nuclei in abnormal cells are generally larger and less sharply delimited when examined microscopically.

When a sample of asynchronous, exponentially growing cells is analyzed on a cell-by-cell basis, we can detect the heterogeneity of the cells due to their different stages of the cell cycle. In fact, single cells exhibit spectral features similar to those observed for pure cell cycle fractions separated by elutriation. Thus, we believe that understanding cell cycle dependent spectra will shed light onto the spectral differences observed previously between normal and abnormal spectra.

\section{CONCLUSIONS}

We present the first ever observation of FTIR spectral features of cells in their different stages of cell division. The observed spectral features are necessary for an understanding of the observed spectral differences between normal and abnormal cells, for the diagnosis of disease. Furthermore, we present a model for the explanation of the absence of DNA spectral features in nuclei of cells that are not actively involved in cell division.

The ML-1 cell line was a gift from Michael Kastan, Department of Oncology, Johns Hopkins University School of Medicine, Baltimore, Maryland. We are grate- 
ful to T. M. Delohery and M. Menon from the Sloan Kettering Cancer Center Core Facility for providing expert cell cycle analysis.

\section{REFERENCES}

1. Chiriboga, L.; Xie, P.; Yee, H.; Vigorita, V.; Zarou, D.; Zakim, D.; Diem, M. Cell Mol Biol 1998, 44, 219-230.

2. Wong, P. T. T.; Wong, R. K.; Caputo, T. A.; Godwin, T. A.; Rigas, B. Proc Natl Acad Sci USA 1991, 88, 10988-10992.

3. Chiriboga, L.; Xie, P.; Yee, H.; Vigorita, V.; Zarou, D.; Zakim, D.; Diem, M. Biospectroscopy 1998, 4, 47-53.

4. Chiriboga, L.; Xie, P.; Yee, H.; Vigorita, V.; Zarou, D.; Zakim, D.; Diem, M. Biospectroscopy 1998, 4, $54-59$.

5. Wood, B. R.; Quinn, M. A.; Burden, F. R.; McNaughton, D. Biospectroscopy 1996, 2, 143-153.

6. Lowry, S. R. Cell Mol Biol 1998, 44, 169-178.

7. Wood, B. R.; Quinn, M. A.; Tait, B.; Ashdown, M.; Hislop, T.; Romeo, M.; McNaughton, D. Biospectroscopy 1998,3 , in press.

8. Malins, D. C.; Polissar, N. L.; Gunselman, S. J. Proc Natl Acad Science USA 1997, 94, 259-264.

9. Fabian, H.; Jackson, M.; Murphy, L.; Watson, P. H.; Fichtner, I.; Mantsch, H. H. Biospectroscopy 1996, 1, 37-46.

10. Hood, L. E.; Wilson, J. H.; Wood, W. B. Molecular Biology of Eukariotic Cells; W.A. Benjamin, Inc.: Menlo Park, CA, 1974.
11. Grant, W. D.; Morrison, M. Anal Biochem 1979, 98, 112-115.

12. Figdor, C. G.; Leemans, J. M.; Bont, W. S.; de Vries, J. E. Cell Biophys 1983, 5, 105-118.

13. McEwen, C. R.; Stallard, R. W.; Juhos, E. T. Anal Biochem 1968, 23, 369-377.

14. Sanderson, R. J.; Bird, K. E.; Palmer, N. F.; Brenman, J. Anal Biochem 1976, 71, 615-622.

15. Crissman, H. A. Darzynkiewicz, Z. Tobey, R. A. Steinkamp, J. A. Science 1985, 228, 1321-1324.

16. Czerniak, B.; Darzynkiewicz, Z.; Herz, F.; Wersto, R. P.; Koss, L. G. Mater Med Pol., 1989, 21, 3-9.

17. Bargonetti, J.; Houser, S. L.; Gopen, T.; Lu, T.; Koshtatyi, S., submitted, 1998.

18. Bruker Optics, Inc., OPUS Spectroscopic Software (OS/2 Version); Billerica, MA, 1997.

19. Diem, M. Introduction to Modern Vibrational Spectroscopy; Wiley-Interscience: New York, 1993.

20. Tsuboi, M.; Takahashi, S.; Harada, I. In Physicochemical Properties of Nucleic Acids; Duchesne, J., Ed.; Academic Press: New York, 1973; Vol. 2, p. 91.

21. Saenger, W. In Principles of Nucleic Acid Chemistry; Cantor, C.R., Ed.; Springer-Verlag: New York, 1984.

22. Benedetti, E.; Bramanti, E.; Papineschi, F.; Rossi, I.; Benedetti, E. Appl Spectroscopy 1997, 51, 792-797.

23. Yazdi, H. M.; Bertrand, M. A.; Wong, P. T. T. Acta Cytologica 1996, 40, 664-668.

24. Benedetti, E.; Bramanti, E.; Papineschi, F.; Vergamini, P.; Benedetti, E. Cell Mol Biol 1998, 44, 129-139.

25. Haaland, D. M.; Jones, H. D. T.; Thomas, E. V. Appl Spectroscopy 1997, 51, 340-345. 